\title{
Thalamic Neuronal Activity in Dopamine-Depleted Primates: Evidence for a Loss of Functional Segregation within Basal Ganglia Circuits
}

\author{
Mathias Pessiglione, ${ }^{1}$ Dominique Guehl, ${ }^{2}$ Anne-Sophie Rolland, ${ }^{1}$ Chantal François, ${ }^{1}$ Etienne C. Hirsch, ${ }^{1}$ Jean Féger, ${ }^{1}$ \\ and Léon Tremblay ${ }^{1}$ \\ ${ }^{1}$ Laboratoire de Neurologie et Thérapeutique Expérimentale, Institut National de la Santé et de la Recherche Médicale Unité 679, Hôpital de la Salpêtrière, \\ 75651 Paris, France, and ${ }^{2}$ Laboratoire de Neurophysiologie, Centre National de la Recherche Scientifique Unité Mixte de Recherche 5542, Université de \\ Bordeaux 2, 33076 Bordeaux, France
}

\begin{abstract}
Different analyses of neuronal activity in primate models of Parkinson's disease (PD) have resulted in two different views on the effects of dopamine depletion. The first is based on the higher firing rate and bursty firing pattern, and assumes that dopamine depletion results in a hyperactivity of basal ganglia (BG) output structures. The second is based on the less-specific responses to passive joint manipulation and the excessive correlations between neuronal discharges, and assumes that dopamine depletion results in a loss of functional segregation in cortico-BG circuits. The aim of the present study was to test out the predictions of these two different views on thalamic neuronal activity. Three male vervet monkeys (Cercopithecus aethiops) were progressively intoxicated with 1-methyl-4-phenyl-1,2,3,6tetrahydropyridine (MPTP). Neuronal activities were characterized using standard analyses (firing rates and patterns, receptive fields, and cross-correlations) and compared between the normal, asymptomatic (before the stabilization of motor symptoms), and parkinsonian (with persistent akinesia and rigidity) stages of MPTP intoxication. The pallidonigral thalamus (receiving projections from the BG) was characterized in both the asymptomatic and parkinsonian states by (1) an unchanged firing rate and pattern and (2) a proliferation of nonspecific neurons and correlated pairs. In contrast, the cerebellar thalamus (receiving projections from the cerebellum), was characterized by no change (asymptomatic state) or minor changes (symptomatic state). Thus the major dysfunction after dopamine depletion appeared to be the loss of functional segregation within cortico-BG circuits, which could also be at the heart of parkinsonian pathophysiology.
\end{abstract}

Key words: monkey; MPTP; Parkinson's disease; dopamine; thalamus; neuronal activity

\section{Introduction}

Parkinson's disease (PD) is characterized clinically by motor symptoms (akinesia, rigidity, and tremor) and histologically by the loss of mesencephalic dopamine (DA) neurons, which principally send their axons to the basal ganglia (BG). Thus, to understand the pathophysiology of PD, we must determine the effects of DA depletion on the information transmitted to the thalamus and cortex by the output structures of the BG, namely the globus pallidus pars internalis (GPi) and the substantia nigra pars reticulata $(\mathrm{SNr})$. Many studies have sought to characterize neuronal activities within these output structures, both in mon-

Received Sept. 30, 2004; revised Dec. 24, 2004; accepted Dec. 28, 2004.

M.P. was supported by the Ministère de la Recherche et de l'Education Nationale (France). D.G. was supported by the Alain Guillard neurological prize. This study was supported by the National Parkinson Foundation (Miami, FL). We thank Arthur Leblois and Thomas Boraud for helpful suggestions on data analysis, Jérôme Yelnik and Dominique Tandé for expert participation in surgical operations and immunohistochemical procedures, and Nicholas Barton for checking English usage.

Correspondence should be addressed to Léon Tremblay, Institut National de la Santé et de la Recherche Médicale Unité 679, Hôpital de la Salpêtrière, 47 Boulevard de l'Hôpital, 75651 Paris cedex 13, France. E-mail: Itremb@ccr.jussieu.fr.

DOI:10.1523/JNEUROSCI.4056-04.2005

Copyright $\odot 2005$ Society for Neuroscience $\quad$ 0270-6474/05/251523-09\$15.00/0 keys rendered parkinsonian by 1-methyl-4-phenyl-1,2,3,6tetrahydropyridine (MPTP) and in PD patients undergoing electrophysiological targeting before lesion or stimulation. Because they analyzed different parameters, these studies reported a variety of changes: (1) an increase in firing rate, (2) a higher incidence of rhythmic and bursty firing patterns, (3) a reduced specificity of responses to passive movements, and (4) excessive correlations between the discharges of distinct neurons (Boraud et al., 2002).

Two theoretical views may explain these changes. According to DeLong (1990), the DA depletion could impair the balance between the direct and indirect pathways linking the striatum to the GPi/SNr, which could account for the increase in firing rate. A high pallidal firing rate may act as a brake on the motor cortex, via the inhibitory projection to the thalamus, resulting in akinesia. According to Bergman et al. (1998), the DA depletion could impair the functional segregation between striatopallidal pathways, explaining both the loss of specificity and the excess of correlations. The inability to decorrelate the BG outputs may lead to coactivations of antagonist motor programs, resulting in both akinesia and rigidity.

Although the effects of MPTP intoxication on pallidal neuronal activity have been investigated extensively, there are yet no 
published data about the following step in cortico-BG circuits, namely the thalamus, except in abstract form (Vitek et al., 1990) and in nonprimate species (Schneider and Rothblat, 1996). The aim of the present study was to fill this gap, to assess which dysfunction is likely to participate in the pathophysiology of PD: the hyperactivity of the BG output structures (predicting a decrease in firing rate) or the loss of functional segregation in the $B G$ circuits (predicting less-specific receptive fields and excessive correlations). Thus, we used standard methods to evaluate all parameters allowing neuronal activities to be characterized: firing rates and patterns, receptive fields, and cross-correlations. To determine which changes were linked to BG dysfunction, we compared two thalamic territories: one receiving inputs from the $\mathrm{GPi} / \mathrm{SNr}$ (referred to as the pallidonigral thalamus) and one receiving inputs from the cerebellum (referred to as the cerebellar thalamus). To determine which changes were linked to the expression of motor symptoms, we compared the normal state with two successive stages of progressive MPTP intoxication: asymptomatic (preceding the stabilization of motor symptoms) and parkinsonian (with persistent rigidity and akinesia). Episodes of resting tremor were excluded from the analysis, because tremorrelated changes in thalamic activities have been described previously (Guehl et al., 2003).

\section{Materials and Methods}

All experiments were performed in accordance with 1996 guidelines specified by the National Institutes of Health and the recommendations contained in the European Community Council Directives (86/609). Three adult male vervet monkeys (Cercopithecus aethiops sabaeus) weighing 6 $7 \mathrm{~kg}$ were used for the study. These monkeys have already been described, and were referred by the same letters (F, R, and G), in behavioral studies reporting early disorders associated with MPTP-induced parkinsonism (Pessiglione et al., 2003, 2004a,b) and in a previous electrophysiological study focusing on the pathophysiology of resting tremor (Guehl et al., 2003). Because the clinical evaluation, schedule of MPTP intoxication, neuronal recording and analysis, and histological procedures are for the most part the same as in previous studies, the methods are only described briefly.

MPTP intoxication. Monkeys were examined daily, both in their home cage and in the recording chair, by an experienced neurologist (D.G.) to rate their clinical state on the scale of Schneider and Kovelowski (1990), ranging from 0 (normal) to 29 (full parkinsonism). In the recording chair, rigidity was assessed by joint manipulation, and tremor was monitored using electromyographic (EMG) recordings. Video recordings of monkeys' behavior were analyzed using Vigie Primates software (Viewpoint, Lyon, France) for items requiring quantification of the number or rapidity of movements (home-cage activity, bradykinesia, eye and arm movements).

Between the normal and the asymptomatic state, monkeys received an initial series of intramuscular MPTP injections $(0.3-0.4 \mathrm{mg} / \mathrm{kg}$ every $4-7$ d) until the clinical score differed from 0 (Table 1). The precise schedule was adapted to each animal to avoid the development of too severe parkinsonism. The neuronal recordings for the asymptomatic state were started only after a period of recovery, allowing the gross motor symptoms to disappear. Between the asymptomatic and the parkinsonian state, the monkeys received a second series of intramuscular MPTP injections $(0.6-0.8 \mathrm{mg} / \mathrm{kg}$ daily), until a severe parkinsonism was stabilized, including persistent akinesia and rigidity. The recording sessions started at least $3 \mathrm{~d}$ after the last injection and lasted 16, 4, and 12 weeks during the normal state, 3,4 , and 3 weeks during the asymptomatic state, and 3, 3, and 3 weeks during the parkinsonian state, respectively, for monkeys F, R, and G. For monkeys F, R, and G, the cumulative doses used were $2.8,1.6$, and $1.2 \mathrm{mg} / \mathrm{kg}$, respectively, to reach the asymptomatic state and 5.3, 2.8, and $3.0 \mathrm{mg} / \mathrm{kg}$, respectively, to reach the parkinsonian state.

Recording techniques. A rectangular stainless-steel recording chamber

\section{Table 1. Clinical scores in the two stages of MPTP-induced parkinsonism}

\begin{tabular}{|c|c|c|c|c|}
\hline \multirow[b]{3}{*}{ Items } & \multicolumn{4}{|c|}{ Clinical state } \\
\hline & \multicolumn{2}{|c|}{ Asymptomatic } & \multicolumn{2}{|c|}{ Parkinsonian } \\
\hline & Start & End & Start & End \\
\hline Home-cage hypokinesia (mild/moderate/severe) & $1,0,1$ & $0,0,0$ & $3,3,3$ & $3,3,3$ \\
\hline Rigidity (mild/moderate/severe) & $0,0,0$ & $0,0,0$ & $3,2,2$ & $2,2,2$ \\
\hline Bradykinesia (mild/moderate/severe) & $0,0,0$ & $0,0,0$ & $3,2,2$ & $3,2,3$ \\
\hline Tremor (mild/moderate/severe) & $0,0,0$ & $0,0,0$ & $3,3,3$ & $2,3,2$ \\
\hline Feeding (reduced/impaired/impossible) & $0,0,0$ & $0,0,0$ & $3,2,3$ & $3,2,3$ \\
\hline Freezing (some/frequent episodes) & $1,0,0$ & $0,0,0$ & $2,2,2$ & $2,2,2$ \\
\hline Posture (some/persistent stooping) & $0,0,0$ & $0,0,0$ & $2,2,2$ & $2,2,2$ \\
\hline Vocalization (reduced/absent) & $0,0,0$ & $0,0,0$ & $2,2,2$ & $2,2,2$ \\
\hline Spontaneous eye movements (few/none) & $1,0,1$ & $0,0,0$ & $2,2,2$ & $2,2,2$ \\
\hline Triggered eye movements (occasional/none) & $0,0,0$ & $0,0,0$ & $1,1,1$ & $2,1,1$ \\
\hline Arm movements (slow/no movements) & $0,0,0$ & $0,0,0$ & $2,1,1$ & $2,1,1$ \\
\hline Arm posture (flexed/dystonic) & $0,0,0$ & $0,0,0$ & $1,1,1$ & $1,1,1$ \\
\hline Total & $3,0,2$ & $0,0,0$ & $27,24,23$ & $27,23,23$ \\
\hline
\end{tabular}

The scores at the start and at the end of the recording periods are given for monkeys $F, R$, and $G$, respectively. They are detailed according to Schneider and Kovelowski (1990), with a total ranging from 0 (normal) to 29 (full parkinsonism).

(32 × $26 \mathrm{~mm}$; Unimécanique, Epinay-sur-Seine, France) was cemented with dental acrylic over the skull of each animal while it was under deep anesthesia (induced by ketamine/atropine injection and maintained by fluothane/halothane inhalation). The anterior commissure (AC) and posterior commissure (PC) were located during the surgical operation by ventriculography. The chamber was positioned stereotactically in the horizontal plane parallel to the AC-PC line, with the center over the interhemispheric line and $2 \mathrm{~mm}$ rostral to the PC. The area of the skull under the chamber was removed, and the dura was left intact. For EMG recordings, Teflon-coated seven-strand stainless-steel wire electrodes were implanted bilaterally into three arm muscles (biceps brachii, triceps brachii, and extensor digitorum). The wire ran subcutaneously from the muscle to a connector cemented close to the recording chamber. Two stainless-steel cylinders allowing head fixation were also cemented in front of and behind the chamber.

The EMG and neuronal activities were recorded simultaneously. The EMG signal collected by the wire electrodes was filtered (passband, 100$1500 \mathrm{~Hz}$ ), amplified (×2000), and digitized at 12,500 Hz (Spike 2; Cambridge Electronic Design, Cambridge, UK). Extracellular activities of single neurons were recorded by glass-insulated and platinum-plated tungsten microelectrodes (impedance of 1.5-6.0 M $\Omega$ at $1000 \mathrm{~Hz}$ ), filtered (passband, $300-3000 \mathrm{~Hz}$ ), and amplified $(\times 10,000)$. For each track, one microelectrode was inserted within subcortical structures through a stainless-steel guide cannula traversing the dura mater. The cannulas were stereotactically positioned within the chamber using a microdrive and arranged in such a way so that the different tracks were at least $1 \mathrm{~mm}$ apart and covered the anteroposterior and mediolateral extension of the pallidonigral and cerebellar thalamus. Isolated waveforms collected by the microelectrodes were passed through a time-voltage template built by the Spike 2 software, to discriminate and produce a pulse for each spike with a temporal resolution of $0.1 \mathrm{~ms}$. When neighboring neurons emit spikes with clearly different forms, the Spike 2 software allows several neurons (maximum of three in practice) to be recorded simultaneously. To avoid a selection bias, we considered all of the neurons with a correct signal-to-noise ratio $(>2: 1)$ encountered along the track.

Two $180 \mathrm{~s}$ acquisition sequences were performed for each neuron or pair of neurons: the first one while the animal was awake and sitting quietly in the chair, and the second one while distal and proximal joints were gently manipulated, one at a time, backward and forward. The receptive fields were detected on-line using loudspeakers and confirmed off-line using neuronal recordings by another experimenter, who was blind to the status of the animal. Tremor was checked both on-line by direct observation and off-line using EMG recordings. Because tremorinduced changes in firing rate and pattern that we have described previously (Guehl et al., 2003) may bias the results, the sequences containing 
Table 2. Comparison of neuronal parameters between the three clinical states

\begin{tabular}{|c|c|c|c|c|}
\hline \multirow[b]{2}{*}{ Parameter } & \multirow[b]{2}{*}{ Territory } & \multicolumn{3}{|l|}{ State } \\
\hline & & Normal & Asymptomatic & Parkinsonian \\
\hline \multirow[t]{2}{*}{ Number of cells } & Pallidonigral (F, R, G) & $257(59,59,139)$ & $189(58,50,81)$ & $235(85,90,60)$ \\
\hline & Cerebellar (F, R, G) & $131(88,27,16)$ & $136(57,29,50)$ & $150(107,16,27)$ \\
\hline \multirow[t]{2}{*}{ Number of pairs } & Pallidonigral (F, R) & $33(15,18)$ & $29(15,14)$ & $38(22,16)$ \\
\hline & Cerebellar (F, R) & $28(15,13)$ & $42(24,18)$ & $51(30,21)$ \\
\hline \multirow[t]{2}{*}{ Firing rate (spikes/s) } & Pallidonigral (F, R, G) & $14.7(16.1,13.1,14.8)$ & $13.8(17.6,13.3,11.5)$ & $15.0(17.4,13.2,14.5)$ \\
\hline & Cerebellar (F, R, G) & $19.7(19.9,16.7,23.7)$ & $17.8(20.1,16.2,16.1)$ & $16.6(16.6,17.4,16.3)$ \\
\hline \multirow[t]{2}{*}{ Bursts (\%) } & Pallidonigral (F, R, G) & $28.1(33.9,33.1,23.5)$ & $32.3(39.0,28.5,29.8)$ & $35.2(32.9,43.9,25.4)$ \\
\hline & Cerebellar (F, R, G) & $30.4(28.4,35.7,32.1)$ & $37.6(35.3,41.4,38.0)$ & $43.5(43.1,48.3,42.2)^{*}$ \\
\hline \multirow[t]{2}{*}{ Specificity (\%) } & Pallidonigral (F, R, G) & $69.4(69.2,65.7,71.1)$ & $39.9(40.1,47.5,35.0)^{*}$ & $14.6(13.3,14.6,16.5)^{*}$ \\
\hline & Cerebellar (F, R, G) & $53.9(54.2,57.5,46.1)$ & $39.4(43.4,38.4,35.5)$ & $28.9(27.9,37.9,27.6)^{*}$ \\
\hline \multirow[t]{2}{*}{ Correlation (\%) } & Pallidonigral (F, R) & $18.1(16.7,19.3)$ & $65.6(70.8,60.1)^{*}$ & $39.6(32.0,50.1)^{*}$ \\
\hline & Cerebellar (F, R) & $21.3(17.7,25.4)$ & $26.1(29.8,21.1)$ & $21.4(21.4,21.4)$ \\
\hline
\end{tabular}

Values are given first for the entire pool of neurons and then for the monkeys $F, R$, and $G$ individually. Bursts, Percentage of firing patterns that contain bursts [both periodic (rhythmic neurons) and not periodic (bursty neurons)]. Specificity, Percentage of neurons responding to one joint only among neurons responding to passive joint movement. Correlation, Percentage of correlated neuronal pairs. Asterisks indicate values significantly different from the normal state.

periods of tremor were eliminated from the analysis of the parkinsonian state.

Data analysis. In addition to the receptive field and the firing rate, the characterization of each neuron was completed by classifying the firing pattern into one of four categories: irregular, regular, bursty, or rhythmic. This classification was made using an algorithm derived from the burst and oscillation detection method proposed by Kaneoke and Vitek (1996). Basically, this method consists of comparing the distribution of discharge density with reference distributions, using a KolmogorovSmirnov test with a significance threshold of $p<0.05$. The firing pattern is termed irregular or regular if the distribution of discharge density is not significantly different from the Poisson or normal distribution, respectively. If the distribution of discharge density is different from both the Poisson and the normal distribution, the algorithm concludes that the spike train contains bursts. In this case, the power spectrum of the spike train is obtained by applying the Fourier transform on the autocorrelogram, calculated for $\pm 1000 \mathrm{~ms}$ offset with a bin size of $5 \mathrm{~ms}$. The firing pattern is considered rhythmic if the power spectrum contains a peak between 3 and $20 \mathrm{~Hz}$ that exceeds the confidence interval of the baseline (with $p<0.01$ ). Otherwise the algorithm concludes that the bursts are not periodic, and the firing pattern is termed bursty.

Correlations were determined using a method inspired by that proposed by Raz et al. (2000). For simultaneously recorded pairs of neurons containing at least 500 spikes each, cross-correlograms were calculated for $\pm 1000 \mathrm{~ms}$ offset with a bin size of $5 \mathrm{~ms}$. The central part of the cross-correlogram $(-10 ; 10 \mathrm{~ms})$ was removed to avoid artifacts caused by the inability to detect overlapping spikes with a single electrode. The pair was considered to be correlated if the cross-correlogram contained a peak exceeding the confidence interval of the baseline (with $p<0.001$ ).

All parameters were expressed as distributions: ranges of firing rate $(0-10 / 10-20 / 20-30 / 30-40 / 40-50 />50$ spikes/s $)$, types of firing pattern (irregular/regular/bursty/rhythmic neurons), specificity level of receptive fields (one joint/one limb/two limbs/more than two limbs), and presence of correlation (correlated/noncorrelated pairs). For each parameter, one dependent variable was chosen for statistical comparisons and is reported in Table 2: the mean firing rate, the percentage of bursting neurons (including bursty and rhythmic patterns), the percentage of specific receptive fields (responding to one joint only), and the percentage of correlated pairs. The significance of differences between the three monkeys, the two thalamic territories, and the three clinical states was determined using a Student's $t$ test for numerical variables (mean firing rate) and a $\chi^{2}$ test for qualitative variables (percentages of bursting neurons, specific receptive fields, and correlated pairs). The $p$ value threshold was set at 0.05 , taking into account corrections required by multiple comparisons.

Histological procedures. At the end of the experiments, the monkeys were killed with an overdose of anesthetic and transcardially perfused with saline followed by fixative solution. The brains were removed from the skull and cut on a freezing microtome into 50- $\mu$ m-thick frontal sections perpendicular to the AC-PC line. Regularly spaced free-floating sections were stained with cresyl violet to identify the electrode tracks and stained for calbindin immunoreactivity to delineate the thalamic territories. Recording locations were reconstructed based on the microdrive coordinates and gliosis associated with guide cannula and microelectrode tracks. The pallidonigral thalamus was identified by clusters of cells that were strongly stained for calbindin immunoreactivity, whereas the cerebellar thalamus was weakly stained (Percheron et al., 1996). In the nomenclature of Olszewski (1952), the pallidonigral thalamus comprises the ventrolateral nucleus pars oralis and the ventral anterior nucleus, whereas the cerebellar thalamus comprises the ventroposterolateral nucleus pars oralis and the more medially located area X. The recording locations and the limits of thalamic territories are illustrated in Figure 1. All neurons located on the border of the thalamus or on the limit between pallidonigral and cerebellar territories were excluded from the analysis.

Regularly spaced free-floating sections were also stained by an immunohistochemical procedure for tyrosine hydroxylase (TH), to control for the degeneration of mesencephalic DA cell groups. Mesencephalic THpositive cells were plotted on nine frontal sections at $1 \mathrm{~mm}$ intervals, using a computer-assisted microscope system (Mercator; Explora Nova, La Rochelle, France). The retrorubral area (A8), substantia nigra pars compacta (A9), and ventral tegmental area (A10) were delineated according to arbitrary outlines as described previously (Herrero et al., 1993). The total number of cells for each subregion was estimated after correction by the Abercrombie (1946) method, and the neuronal loss was evaluated with reference to average values obtained in three intact male vervet monkeys (Francois et al., 1999).

\section{Results}

\section{Clinical evaluation}

At the end of the first series of MPTP injections, the clinical scores were 3,17 , and 18 for monkeys F, R, and G, respectively. After the subsequent period of recovery, clinical signs were limited to a mild reduction in home-cage activity and spontaneous eye movements, with occasional episodes of freezing (Table 1). Thus, the asymptomatic state, corresponding to a clinical score of between 0 and 3 in all monkeys, was characterized by a mild hypokinesia and by the absence of gross motor symptoms such as akinesia, rigidity, or tremor. At the end of the second series of MPTP injections, the clinical scores were 26, 23, and 24 for monkeys F, $\mathrm{R}$, and $\mathrm{G}$, respectively. These scores were maintained between 23 and 27 throughout the parkinsonian state, which was thus characterized by persistent akinesia, rigidity and postural disorders, and intermittent episodes of resting tremor. TH immunohistochemistry confirmed the severity of the dopaminergic lesion: neuronal loss was 53,60, and $64 \%$ in A8; 82,88 , and $88 \%$ in A9; and 29,45 , and $49 \%$ in A10 for monkeys F, R, and G, respectively. 


\section{Neuronal database}

In total, 1098 neurons were recorded. The relative contribution of each animal varied slightly with the clinical state: for monkeys $F$, $R$, and $G$, respectively, the number of recorded neurons was 147, 86, and 155 in the normal state, 115,79 , and 131 in the asymptomatic state, and 192, 106, and 87 in the parkinsonian state. The absence of any significant difference in firing rates and patterns was checked for each clinical state before data for the three monkeys were pooled. Among the 388, 325, and 385 neurons representing the normal, asymptomatic, and parkinsonian states, 122,142 , and 178 were recorded by pairs, respectively. The sampling of thalamic territories was similar in the three clinical states $(\mathrm{Ta}$ ble 2). Recording locations were distributed between $\mathrm{PC}+2.3 \mathrm{~mm}$ and $\mathrm{PC}+7.3$ $\mathrm{mm}$ along the anteroposterior axis and between 1 and $7 \mathrm{~mm}$ from the interhemispheric line along the mediolateral axis (Fig. 1). Based on calbindin sections, the cerebellar thalamus was delineated between $\mathrm{PC}+2.3 \mathrm{~mm}$ and $\mathrm{PC}+4.3 \mathrm{~mm}$, and the pallidonigral thalamus was delineated between $\mathrm{PC}+4.3 \mathrm{~mm}$ and $\mathrm{PC}+7.3 \mathrm{~mm}$.

\section{Parameters of neuronal activities}

Firing rates

The evolution of firing rates with MPTP intoxication is shown in Figure 2. Whatever the clinical state, the firing rate was extremely varied in both the pallidonigral and the cerebellar territory, as shown by the wide range of values (from 0.2 to 75.1 spikes/s) and by the SDs (12.8-13.7 spikes/s). Compared with the normal state, the firing rate was unchanged in the parkinsonian state, in both the pallidonigral thalamus (14.7 vs 15.0 spikes/s) and the cerebellar thalamus (19.7 vs 16.6 spikes/s). In both territories, the median was between 1.2 and 2.9 spikes/s under the mean, whatever the clinical state.

\section{Firing patterns}

The evolution of firing patterns with MPTP intoxication is shown in Figure 3. In the normal state, the firing patterns were predominantly irregular $(67.8-61.5 \%)$, rarely regular $(4.3-7.7 \%$ of neurons), and sometimes bursty (14.3-13.8\%) or rhythmic (13.6-16.9\%) in both the pallidonigral and cerebellar territories. The bursts typically contained 3 to 10 spikes, and the rhythms were within the range of tremor frequency, between 3 and $8 \mathrm{~Hz}$ (Fig. 3A). In the asymptomatic state, the percentage of neurons with bursts (rhythmic or not) was not significantly modified (Fig. $3 B$ ). In the parkinsonian state, this percentage was also unchanged in the pallidonigral thalamus (28.1 and $35.2 \%$ ) but was increased in the cerebellar thalamus
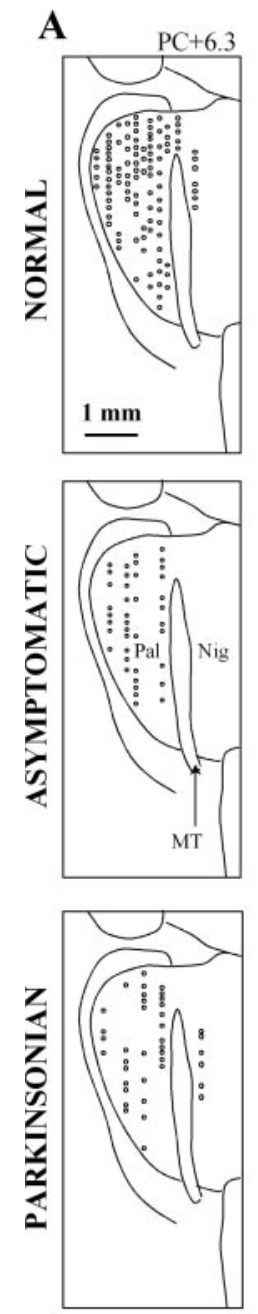
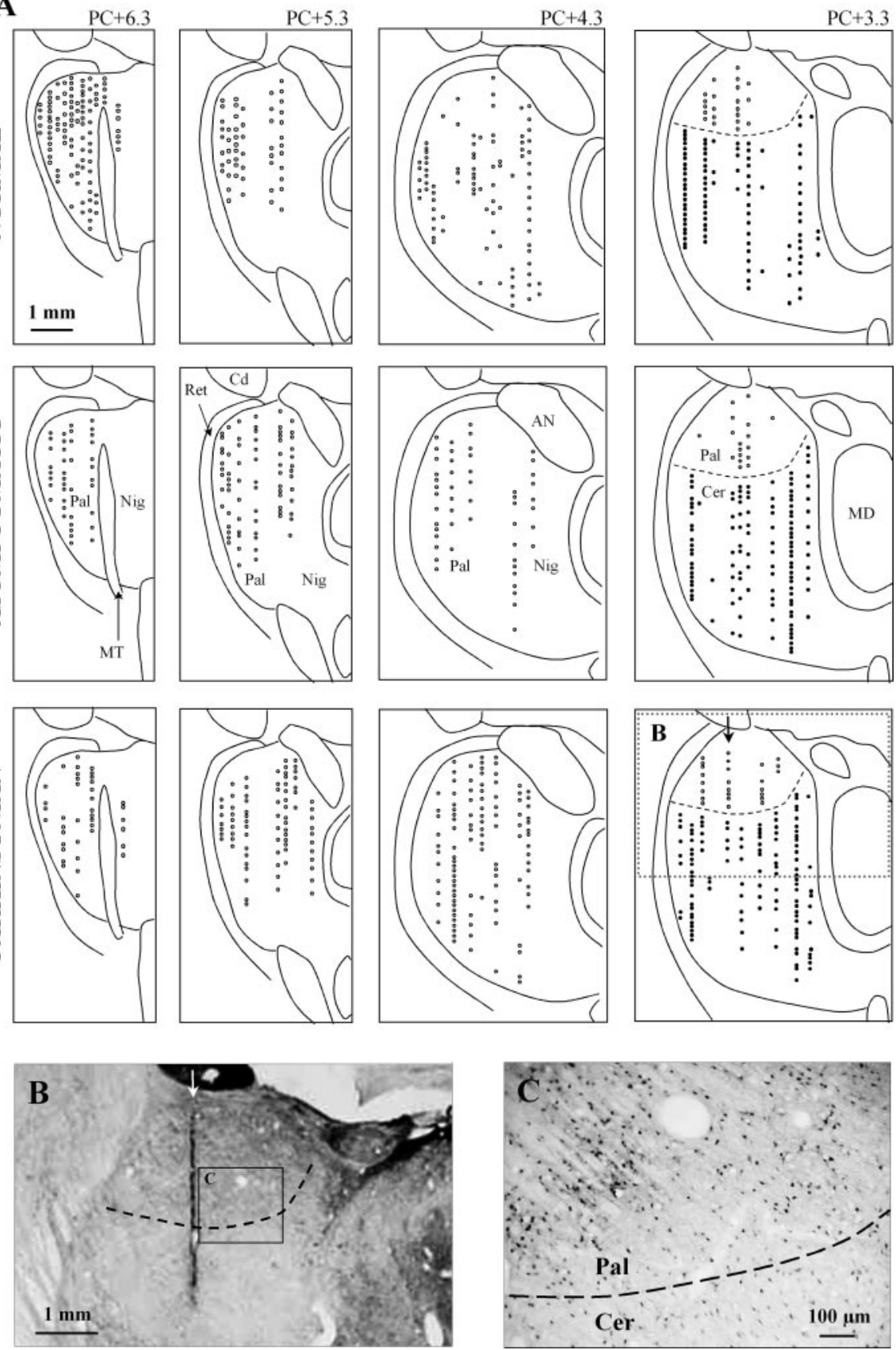

Figure 1. Anatomical localization of recording sites in the three clinical states. A, Mapping of all sites recorded in the two hemispheres of the three monkeys, pooled on four frontal planes ranging from +3.3 to $+6.3 \mathrm{~mm}$ from the $\mathrm{PC}$. The border (dashed line) between the pallidonigral (open circles) and the cerebellar (filled circles) thalamus appears only on the last plane (right column). The pallidal thalamus is more lateral than the nigral thalamus, but the border between these two territories remains hard to delineate. $B$, Example of a calbindin-stained section with a highly visible trace of an electrode track (arrow) corresponding to the last recordings in the parkinsonian state. $C$, Enlarged view of the boxed section in $B$ showing the border between the pallidal and cerebellar thalamus. Only the pallidal thalamus contains black-stained neurons. AN, Anterior nucleus; Cer, cerebellar thalamus; Cd, caudate nucleus; MD, mediodorsal nucleus; MT, mamillothalamic tract; Nig, nigral thalamus; Pal, pallidal thalamus; Ret, reticular nucleus. (from 30.4 to $43.5 \% ; p<0.05$ ), to the detriment of both regular and irregular neurons.

\section{Receptive fields}

The evolution of receptive fields with MPTP intoxication is shown in Figure 4. Analysis of the specificity of receptive fields was performed on 112, 111, and 127 responsive neurons (i.e., 

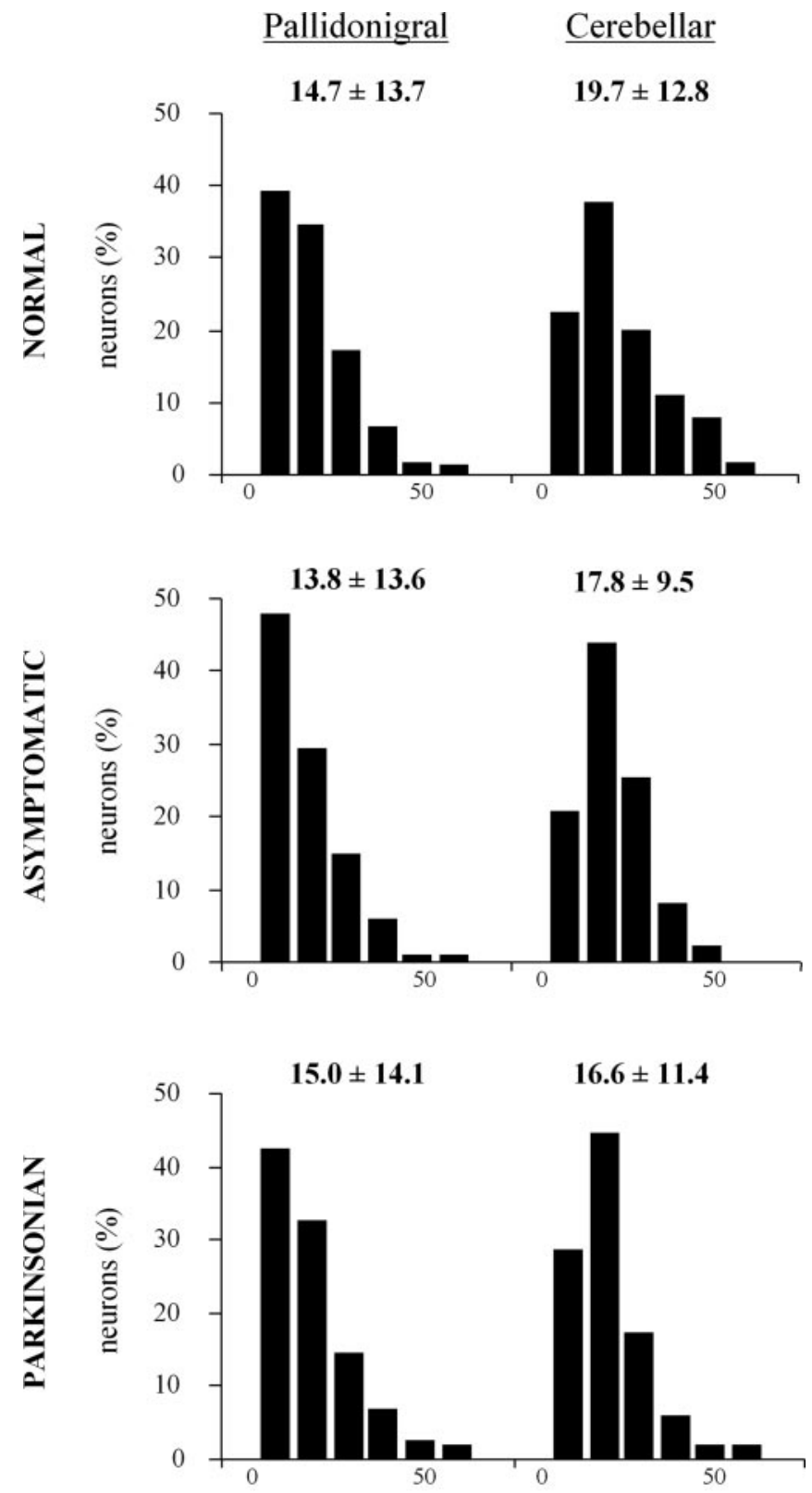

Firing rate (spikes/s)

Figure 2. Evolution of firing rates with MPTP intoxication. Values are means \pm SD. Bars indicate the percentage of neurons with a firing rate within each of the following six ranges: $0-10,10-20,20-30,30-40,40-50$, and $>50$ spikes/s. Data of the three monkeys were pooled.

those that responded to at least one passive joint movement) for the normal, asymptomatic, and parkinsonian states, respectively. In the normal state, the majority of responsive neurons were specific to one joint in both the pallidonigral and the cerebellar thalamus (69.4 and 53.9\%, respectively). The percentage of responsive neurons was not significantly changed in the asymptomatic state. In the parkinsonian state, the percentage was increased in the pallidonigral thalamus (from 24.1 to $32.0 \%$; $p<$ 0.05 ) but not in the cerebellar thalamus (38.1 and 34.7\%). The percentage of specific neurons (responding to one joint only) was decreased in the pallidonigral thalamus (from 69.4 to $39.9 \%$; $p<$ 0.01 ) but was not significantly changed in the cerebellar thalamus (53.9 and $39.4 \%$ ) in the asymptomatic state. In the parkinsonian state, it was decreased in both the pallidonigral thalamus (from 69.4 to $14.6 \% ; p<0.001$ ) and the cerebellar thalamus (from 53.9 to $28.9 \% ; p<0.05$ ). Nonspecific neurons could respond to several joints of a single limb, to the lower and upper limbs of the same side, or to both ipsilateral and contralateral limbs (Fig. 4A). All of these types of nonspecific neurons increased in proportion to MPTP intoxication (Fig. 4B).

\section{Cross-correlations}

The evolution of cross-correlations with MPTP intoxication is shown in Figure 5. Correlations between neuronal discharges were assessed between neighboring neurons recorded by a single electrode. Correlated discharges were typically characterized on the spike train by the temporal correspondence between pauses and on the cross-correlogram by a large positive peak around a time lag of $0 \mathrm{~ms}$, without any oscillation (Fig. $5 \mathrm{~A}$ ). In the normal state, the percentage of correlated pairs was 18.1 and $21.3 \%$ in the pallidonigral and cerebellar thalamus, respectively (Fig. 5B). This percentage was increased in the pallidonigral thalamus, both in the asymptomatic state (from 18.1 to $65.6 \%$; $p<0.001$ ) and in the parkinsonian state (from 18.1 to $39.6 \%$; $p<0.05$ ), whereas it remained approximately the same in the cerebellar thalamus.

\section{Interactions between neuronal parameters}

Data for the three clinical states (normal, asymptomatic, and parkinsonian) are given below.

\section{Interaction with the firing rate}

The firing rate was similar for irregular neurons $(15.7,14.6$, and 15.2 spikes/s) and neurons with bursts (15.6, 15.9, and 16.4 spikes/s) for the normal, asymptomatic, and parkinsonian states, respectively. Regular neurons had a higher firing rate, which tended to decrease $(29.2,24.9$, and 22.1 spikes/s, respectively), but they grew rare with MPTP intoxication. Considering receptive fields, the firing rate was similar for specific neurons (16.1, 15.3, and 20.1 spikes/s, respectively) and nonspecific neurons (18.3, 16.0, and 16.3 spikes/s, respectively). Considering crosscorrelations, the firing rate was also similar for correlated pairs (15.8, 13.2, and $15.2 \mathrm{spikes} / \mathrm{s}$, respectively) and noncorrelated pairs $(16.4,15.7$, and 15.5 spikes/s, respectively).

\section{Interaction with the firing pattern}

The above analysis deals only with irregular neurons and neurons with bursts (rhythmic or not rhythmic), because regular neurons were too rare to be divided into subcategories. For the normal, asymptomatic, and parkinsonian states, the proportion of specific receptive fields was similar between irregular neurons (62.6, 35.9 , and $23.8 \%$, respectively) and neurons with bursts (64.6, 39.9 , and $23.0 \%$, respectively). Thus, the loss of specificity with MPTP intoxication followed the same course for both types of neurons. For cross-correlations, only the pallidonigral thalamus was taken into account, because very few correlated pairs remained in the cerebellar thalamus. The proportion of correlated pairs was $37.4,93.5$, and $43.2 \%$ for pairs including at least one neuron with bursts and $8.2,56.0$, and $38.5 \%$ for pairs including at least one irregular neuron. Thus, bursting activities were more frequently correlated than irregular ones in the normal and asymptomatic states, but not in the parkinsonian state. Hence the excess of correlations was attributable to both types of neurons in the asymptomatic state, whereas it was only attributable to irregular neurons in the parkinsonian state.

\section{Discussion}

In both the asymptomatic and parkinsonian states of MPTPinduced DA depletion, neuronal activities of the pallidonigral 
thalamus were characterized by (1) no change in firing rate, (2) no change in firing pattern, (3) a decreased specificity of receptive fields, and (4) a higher proportion of correlated pairs. The paragraphs below compare these results with the predictions of two dominant views of BG dysfunction in $\mathrm{PD}$, based on either the hyperactivity of the BG output structures or the loss of functional segregation in the BG circuits.

\section{What results from the hyperactivity of} the BG output structures?

Several single-unit studies of MPTPintoxicated monkeys reported both an increased firing rate and a higher proportion of bursting neurons in the GPi (Filion and Tremblay, 1991; Boraud et al., 1998) and to a lesser extent in the $\mathrm{SNr}$ (Wichmann et al., 1999). The hyperactivity of the GPi/ $\mathrm{SNr}$ is thought to put a brake on cortical activity through the inhibitory projection to the thalamus (Alexander and Crutcher, 1990; DeLong, 1990). The prediction was that the pallidonigral thalamus is inhibited by the high and bursting firing of pallidonigral neurons.

In line with previous studies of singleunit thalamic activities (Anderson and Turner, 1991; Vitek et al., 1994), we observed in the normal state a wide range of firing rates (15-20 spikes/s on average) and a predominance of irregular firing patterns (62-68\% of neurons). MPTP injections did not change the firing rate and pattern in the pallidonigral thalamus, which thus cannot be considered as a mere relay of the BG outputs. Indeed, thalamic processes are more complex, because of the high proportion of inhibitory interneurons (25-30\%) and the multiple afferent projections from the frontal cortex, the reticular nucleus, and different modulatory systems (Ilinsky and Kultas-Ilinsky, 2002). The firing rate may have been decreased in some neurons (possibly the projection neurons) and equally increased in other neurons (possibly the interneurons), but such a situation would be likely to result in a bimodal distribution, which we did not observe. Our findings are consistent with neuronal recordings of MPTPintoxicated monkeys that showed no modification of firing rate in the primary motor cortex (Goldberg et al., 2002). However, these findings do not rule out the possibility that thalamic neurons could be less activated when a movement is to be made, as shown by cortical neurons (Escola et al., 2003). Yet our data do not match the prediction that the hyperactivity of the BG output structures results in a reduction of thalamic activity. They also indicate that the firing pattern may not be modified by MPTP intoxication in the absence of tremor. It is noteworthy that the incidence of rhythmic bursts would have been higher if periods of
A

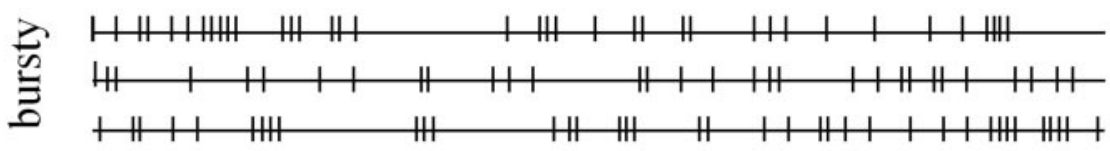

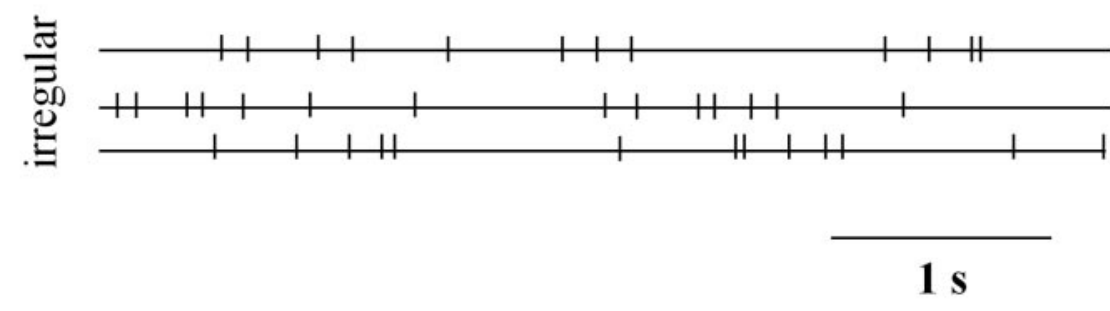

B

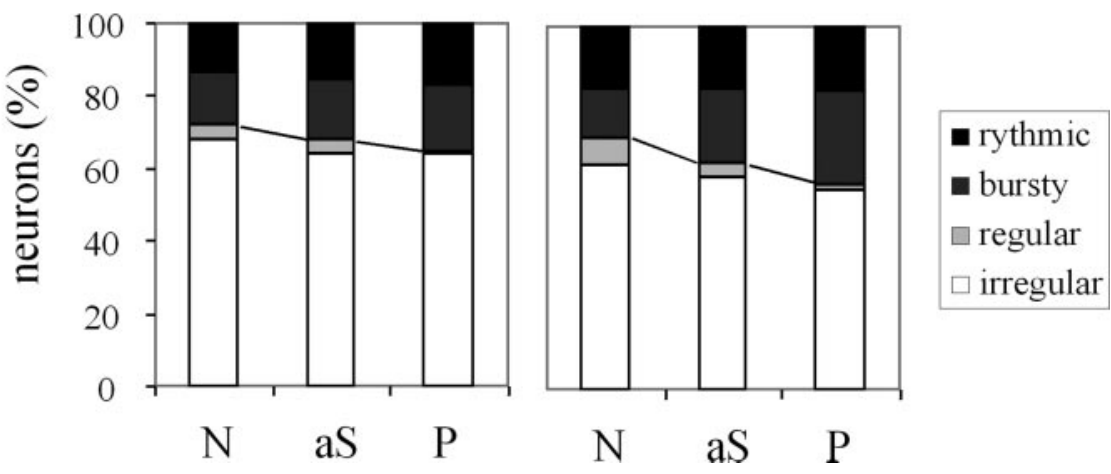

Figure 3. Evolution of firing patterns with MPTP intoxication. $A$, Examples of the four firing patterns that were characterized $B$, Distribution of the different firing patterns. $A \chi^{2}$ test was performed to compare the percentage of neurons with bursts (regardless of whether or not they were rhythmic) between two successive clinical states. No significant difference was found. N, Normal; aS, asymptomatic; P, parkinsonian. Data of the three monkeys were pooled.

resting tremor had been included in the analysis, as we have reported previously (Guehl et al., 2003).

\section{What results from the loss of functional segregation in the BG circuits?}

Several studies in MPTP-intoxicated monkeys reported a decreased specificity of pallidal responses to passive joint manipulation or to striatal microstimulation (Filion et al., 1988; Tremblay et al., 1989; Boraud et al., 2000) and excessive correlations between pallidal discharges (Nini et al., 1995; Raz et al., 2000). These changes are thought to reveal a loss of functional segrega- 
A

\section{NORMAL ASYMPTOMATIC}

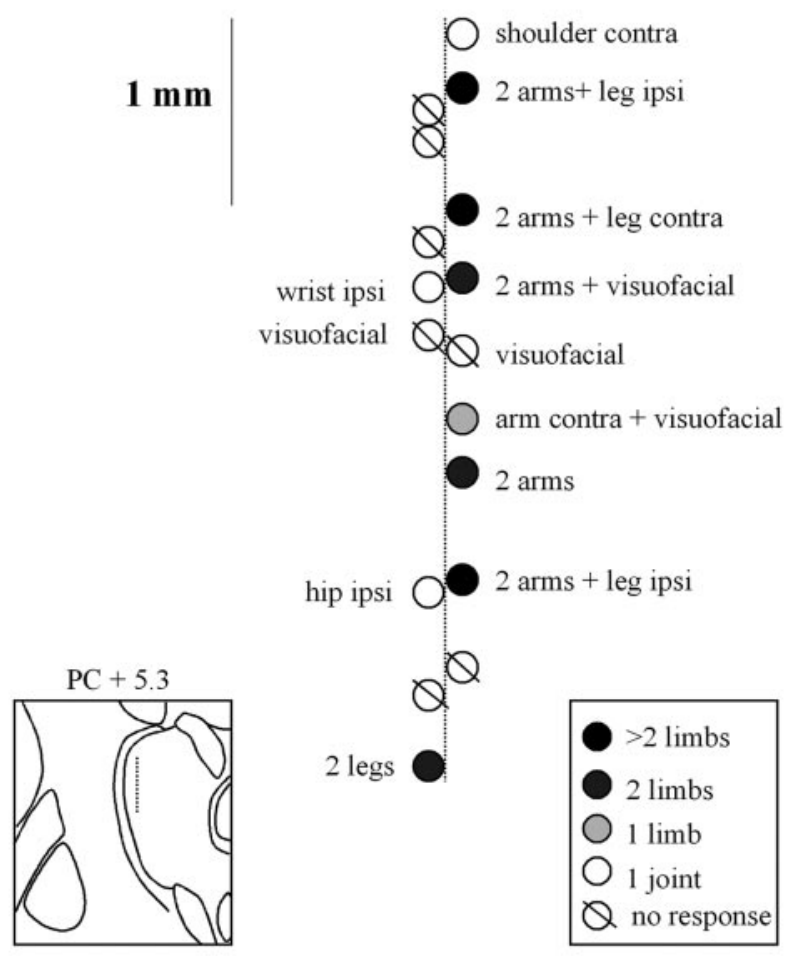

B

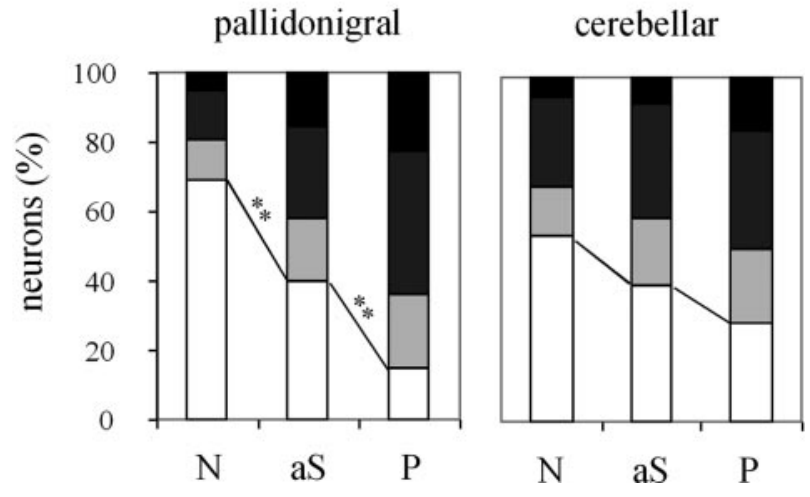

Figure 4. Evolution of receptive fields with MPTP intoxication. A, Examples of receptive fields characterized along an electrode track in the normal and asymptomatic states. The two tracks were made in the pallidonigral thalamus of the same monkey and positioned at the same stereotactic coordinates within the recording chamber, as illustrated on the frontal section. Dorsoventral coordinates of neurons (circles) are in proportion to their position on the vertical axis. Neurons that did not respond to joint manipulation are shown as slashed circles. contra, Contralateral; ipsi, ipsilateral. $B$, Distribution of the different levels of specificity. A $\chi^{2}$ test was performed to compare the percentage of specific neurons (responding to one joint only) between two successive clinical states. ${ }^{* *} p<0.01$. N, Normal; aS, asymptomatic; P, parkinsonian. The data of the three monkeys were pooled.

tion in the BG circuits (Bergman et al., 1998). The prediction was that the loss of specificity and the excess of correlations may be transmitted to the pallidonigral thalamus.

In line with previous studies of thalamic single-unit activities (Butler et al., 1992; Vitek et al., 1994), we observed that responses of thalamic neurons to passive manipulation were specific to one joint in a majority of cases (54-69\%). After MPTP intoxication, the specificity of receptive fields was markedly decreased (from 69 to $15 \%$ ) in the pallidonigral thalamus, with neurons frequently responding to manipulation of several joints and even several limbs. Interestingly, this decrease in specificity appeared in the asymptomatic state, which rules out the possibility that it would merely be a secondary effect of muscular rigidity. A similar result was reported both in the primary motor cortex (Goldberg et al., 2002) and in the supplementary motor area (Escola et al., 2002), with an absence of correlation between the level of specificity and the clinical score for rigidity. The latter study failed to show a decrease in specificity in the presymptomatic state, but this state is likely to be less advanced than our asymptomatic state, which occurred after recovery from mild motor symptoms. In the symptomatic state, we also observed a decreased specificity of receptive fields in the cerebellar thalamus, which must involve other neuronal systems, because the cerebellar thalamus is not considered to receive direct projections from the GPi/SNr (Hirai and Jones, 1989; Percheron et al., 1996). One possibility is that the loss of functional segregation that occurred in the BG may have been propagated to the cerebellar thalamus through corticocerebellar loops. Another possibility is that the decrease in specificity was caused by the DA depletion occurring within the thalamus itself in the parkinsonian state (Freeman et al., 2001).

A previous study used single-unit recordings to test crosscorrelations (Bar-Gad et al., 2003) and reported that pallidal neurons were rarely correlated in the normal state $(<10 \%)$. We also obtained a moderate proportion (18-21\%) of correlated pairs at the thalamic level in the normal state. This proportion was markedly increased in the asymptomatic state (from 18 to 66\%) only for the pallidonigral thalamus, in the absence of any change in firing rate or pattern. This dissociation is noteworthy, because cross-correlograms are highly dependent on both the firing rate and pattern of the neurons constituting the pair (Bar-Gad et al., 2001). A similar dissociation has been observed using multiunit recordings in both the GPi and the primary motor cortex of a nontremulous MPTP-intoxicated monkey (Goldberg et al., 2002). This argues against the interpretation that synchronization of neighboring neurons only reflects increased local interactions. In our study, the excessive correlations were attributable to both irregular and bursting neurons in the asymptomatic state but only to irregular neurons in the parkinsonian state. This could again be explained by the exclusion of tremor episodes, which is likely to exclude periods of synchronized bursting. Indeed, we previously described the occurrence of rhythmic bursts during tremor in both the pallidonigral and cerebellar thalamus (Guehl et al., 2003). Tremor may therefore occur when correlated activities turn into synchronized oscillations that spread over the cerebellar thalamus. Such an interpretation is consistent with studies of PD patients showing that pallidal synchronized oscillations are limited to tremor episodes (Levy et al., 2002), that tremor-related oscillations occur in the cerebellar thalamus (Lenz et al., 1988), and that tremor is improved by highfrequency stimulation of the cerebellar thalamus (Koller et al., 2000).

\section{From BG dysfunction to PD symptoms}

In conclusion, the major BG dysfunction after dopamine depletion could be the loss of functional segregation, which appeared to affect only the pallidonigral thalamus in the asymptomatic state, and to extend to the cerebellar thalamus with the appearance of parkinsonian symptoms. This dysfunction may impair the processes involving at least the motor and cognitive circuits, because motivational processes were shown to be preserved 
throughout MPTP intoxication (Pessiglione et al., 2004b), in accordance with the relative preservation of DA fibers in the limbic parts of the BG, as reported both in parkinsonian monkeys (Jan et al., 2003) and in PD patients (Kish et al., 1988). For instance, the inability to decorrelate motor subcircuits may explain why PD patients have difficulty in performing two simultaneous movements (Benecke et al., 1986; Castiello et al., 1993), and the inability to decorrelate cognitive subcircuits could explain why they are particularly impaired in double-task paradigms (Brown and Marsden, 1991; Malapani et al., 1994). With the progression of the disease, the loss of functional segregation may first induce interferences within the motor or cognitive circuit and then between motor and cognitive subcircuits. During MPTP intoxication, we observed an impairment of movement selection in the presymptomatic state (Pessiglione et al., 2003) and some executive deficits with mild parkinsonism (such as hesitations and freezing) attributable to the combination of motor and cognitive difficulties (Pessiglione et al., 2004a). Interferences between cognitive and motor processes could also account for the loss of temporal uncoupling between mental deliberation and motor execution, which is observed in PD patients making a decision (M. Pessiglione and L. Tremblay, unpublished observations). For the sake of speculation, one can try to draw a connection between the loss of functional segregation and the clinical symptoms of PD. Within the motor circuit, the loss of functional segregation could lead to coselection of antagonist motor programs, resulting in muscular rigidity or in tremor when correlated discharges turn into synchronized oscillations. Within the cognitive circuit, the loss of functional segregation could impair some executive functions, such as the ability to select, maintain, or change a cognitive program depending on the context, a known impairment in DA-depleted PD patients (Cools et al., 2001). With regard to akinesia, the question of whether it results from a dysfunction of the motor or the cognitive circuit, or from interferences between the two, remains open.

\section{References}

Abercrombie M (1946) Estimation of nuclear population from microtome sections. Anat Rech 94:239-247.

Alexander GE, Crutcher MD (1990) Functional architecture of basal ganglia circuits: neural substrates of parallel processing. Trends Neurosci 13:266-271.

Anderson ME, Turner RS (1991) Activity of neurons in cerebellar-receiving and pallidal-receiving areas of the thalamus of the behaving monkey. J Neurophysiol 66:879-893.

$\mathbf{A}$

B

\section{NORMAL}
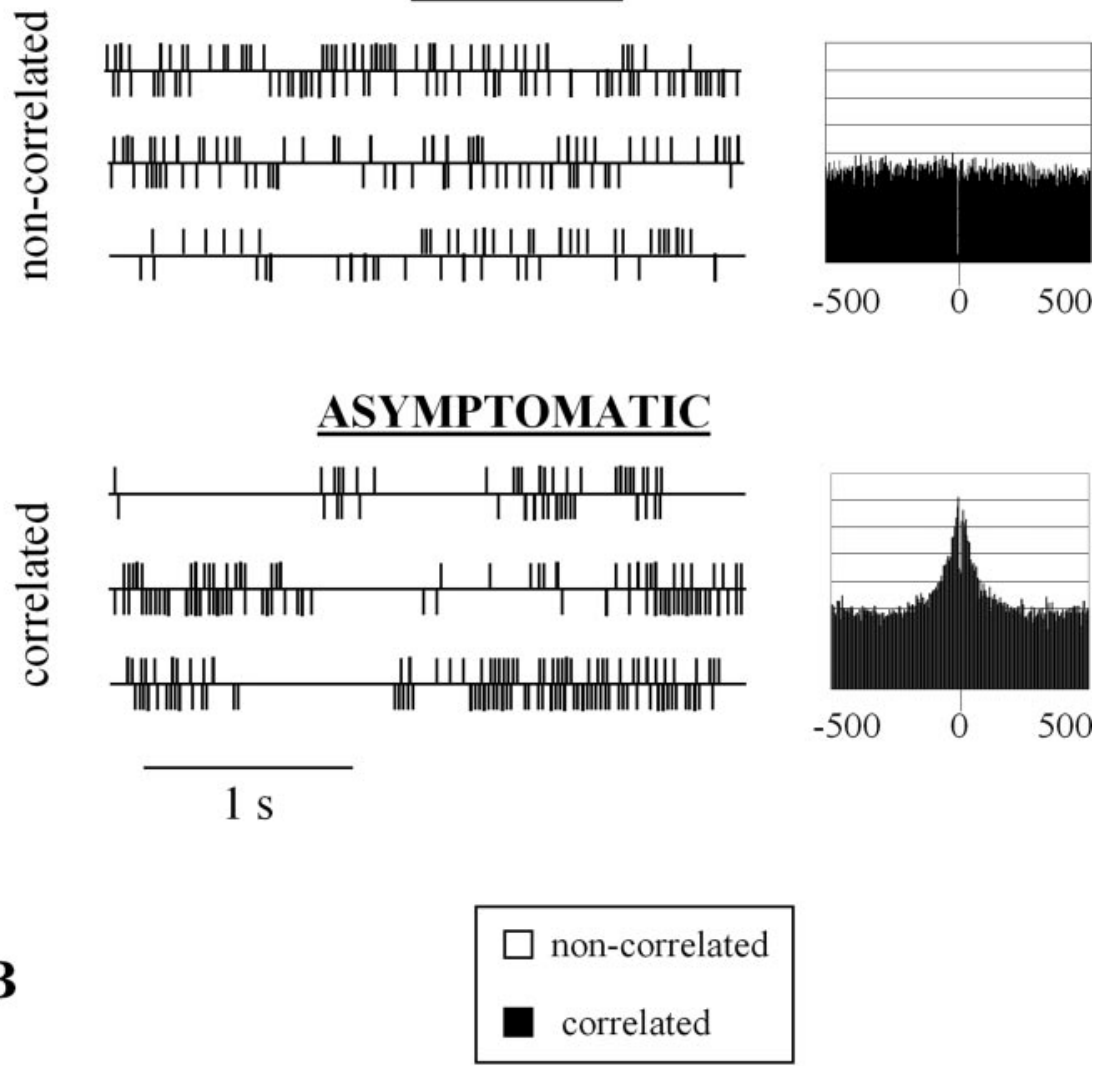

pallidonigral

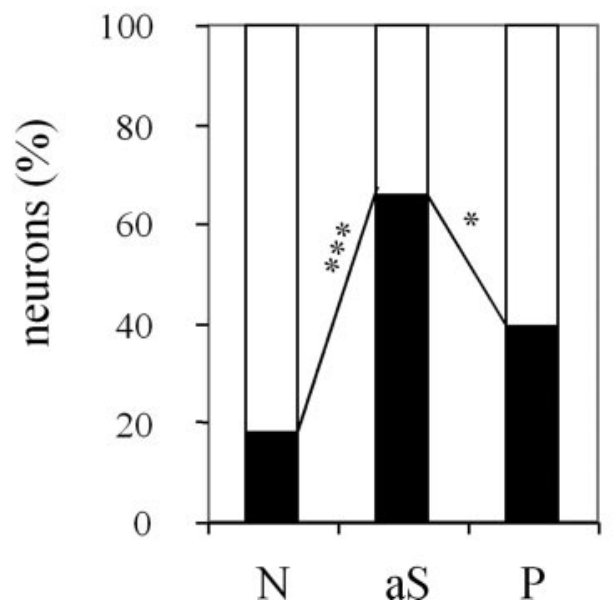

cerebellar

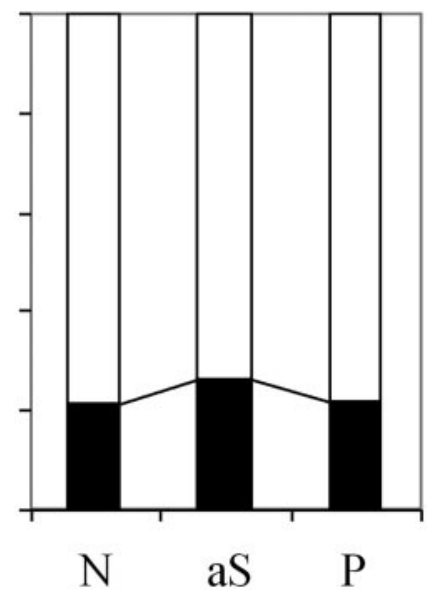

Figure 5. Evolution of cross-correlations with MPTP intoxication. A, Examples of spike trains and cross-correlograms for noncorrelated and correlated pairs recorded in the normal and asymptomatic states. The two recordings were made in the pallidonigral thalamus of the same monkey. The two unit activities are displayed above and below the horizontal line. For the cross-correlograms, the $y$-axis is in arbitrary units and the $x$-axis is in milliseconds. $B$, Distribution of correlated and noncorrelated pairs. $A \chi^{2}$ test was performed to compare the percentage of correlated pairs between two successive clinical states. ${ }^{*} p<0.05$; ${ }^{* * *} p<0.001$. N, Normal; aS, asymptomatic; P, parkinsonian. The data of the three monkeys were pooled.
Bar-Gad I, Ritov Y, Vaadia E, Bergman H (2001) Failure in identification of overlapping spikes from multiple neuron activity causes artificial correlations. J Neurosci Methods 107:1-13.

Bar-Gad I, Morris G, Bergman H (2003) Information processing, dimensionality reduction and reinforcement learning in the basal ganglia. Prog Neurobiol 71:439-473. 
Benecke R, Rothwell JC, Dick JP, Day BL, Marsden CD (1986) Performance of simultaneous movements in patients with Parkinson's disease. Brain 109:739-757.

Bergman H, Feingold A, Nini A, Raz A, Slovin H, Abeles M, Vaadia E (1998) Physiological aspects of information processing in the basal ganglia of normal and parkinsonian primates. Trends Neurosci 21:32-38.

Boraud T, Bezard E, Guehl D, Bioulac B, Gross C (1998) Effects of L-DOPA on neuronal activity of the globus pallidus externalis (GPe) and globus pallidus internalis (GPi) in the MPTP-treated monkey. Brain Res 787:157-160

Boraud T, Bezard E, Bioulac B, Gross CE (2000) Ratio of inhibited-toactivated pallidal neurons decreases dramatically during passive limb movement in the MPTP-treated monkey. J Neurophysiol 83:1760-1763.

Boraud T, Bezard E, Bioulac B, Gross CE (2002) From single extracellular unit recording in experimental and human Parkinsonism to the development of a functional concept of the role played by the basal ganglia in motor control. Prog Neurobiol 66:265-283.

Brown RG, Marsden CD (1991) Dual task performance and processing resources in normal subjects and patients with Parkinson's disease. Brain 114:215-231

Butler EG, Horne MK, Rawson JA (1992) Sensory characteristics of monkey thalamic and motor cortex neurones. J Physiol (Lond) 445:1-24.

Castiello U, Stelmach GE, Lieberman AN (1993) Temporal dissociation of the prehension pattern in Parkinson's disease. Neuropsychologia 31:395-402.

Cools R, Barker RA, Sahakian BJ, Robbins TW (2001) Enhanced or impaired cognitive function in Parkinson's disease as a function of dopaminergic medication and task demands. Cereb Cortex 11:1136-1143.

DeLong MR (1990) Primate models of movement disorders of basal ganglia origin. Trends Neurosci 13:281-285.

Escola L, Michelet T, Douillard G, Guehl D, Bioulac B, Burbaud P (2002) Disruption of the proprioceptive mapping in the medial wall of parkinsonian monkeys. Ann Neurol 52:581-587.

Escola L, Michelet T, Macia F, Guehl D, Bioulac B, Burbaud P (2003) Disruption of information processing in the supplementary motor area of the MPTP-treated monkey: a clue to the pathophysiology of akinesia? Brain 126:95-114.

Filion M, Tremblay L (1991) Abnormal spontaneous activity of globus pallidus neurons in monkeys with MPTP-induced parkinsonism. Brain Res 547:142-151

Filion M, Tremblay L, Bedard PJ (1988) Abnormal influences of passive limb movement on the activity of globus pallidus neurons in parkinsonian monkeys. Brain Res 444:165-176.

Francois C, Yelnik J, Tande D, Agid Y, Hirsch EC (1999) Dopaminergic cell group A8 in the monkey: anatomical organization and projections to the striatum. J Comp Neurol 414:334-347.

Freeman A, Ciliax B, Bakay R, Daley J, Miller RD, Keating G, Levey A, Rye D (2001) Nigrostriatal collaterals to thalamus degenerate in parkinsonian animal models. Ann Neurol 50:321-329.

Goldberg JA, Boraud T, Maraton S, Haber SN, Vaadia E, Bergman H (2002) Enhanced synchrony among primary motor cortex neurons in the 1-methyl-4-phenyl-1,2,3,6-tetrahydropyridine primate model of Parkinson's disease. J Neurosci 22:4639-4653.

Guehl D, Pessiglione M, Francois C, Yelnik J, Hirsch EC, Feger J, Tremblay L (2003) Tremor-related activity of neurons in the "motor" thalamus: changes in firing rate and pattern in the MPTP vervet model of parkinsonism. Eur J Neurosci 17:2388-2400.

Herrero MT, Hirsch EC, Kastner A, Ruberg M, Luquin MR, Laguna J, JavoyAgid F, Obeso JA, Agid Y (1993) Does neuromelanin contribute to the vulnerability of catecholaminergic neurons in monkeys intoxicated with MPTP? Neuroscience 56:499-511.

Hirai T, Jones EG (1989) A new parcellation of the human thalamus on the basis of histochemical staining. Brain Res Brain Res Rev 14:1-34.

Ilinsky IA, Kultas-Ilinsky K (2002) Motor thalamic circuits in primates with emphasis on the area targeted in treatment of movement disorders. Mov Disord 17 [Suppl 3]:S9-S14.

Jan C, Pessiglione M, Tremblay L, Tande D, Hirsch EC, Francois C (2003) Quantitative analysis of dopaminergic loss in relation to the functional territories in MPTP-treated monkeys. Eur J Neurosci 18:2082-2086.

Kaneoke Y, Vitek JL (1996) Burst and oscillation as disparate neuronal properties. J Neurosci Methods 68:211-223.

Kish SJ, Shannak K, Hornykiewicz O (1988) Uneven pattern of dopamine loss in the striatum of patients with idiopathic Parkinson's disease. Pathophysiologic and clinical implications. N Engl J Med 318:876-880.

Koller WC, Pahwa PR, Lyons KE, Wilkinson SB (2000) Deep brain stimulation of the Vim nucleus of the thalamus for the treatment of tremor. Neurology 55:S29-S33.

Lenz FA, Tasker RR, Kwan HC, Schnider S, Kwong R, Murayama Y, Dostrovsky JO, Murphy JT (1988) Single unit analysis of the human ventral thalamic nuclear group: correlation of thalamic "tremor cells" with the 3-6 Hz component of parkinsonian tremor. J Neurosci 8:754764

Levy R, Hutchison WD, Lozano AM, Dostrovsky JO (2002) Synchronized neuronal discharge in the basal ganglia of parkinsonian patients is limited to oscillatory activity. J Neurosci 22:2855-2861.

Malapani C, Pillon B, Dubois B, Agid Y (1994) Impaired simultaneous cognitive task performance in Parkinson's disease: a dopamine-related dysfunction. Neurology 44:319-326.

Nini A, Feingold A, Slovin H, Bergman H (1995) Neurons in the globus pallidus do not show correlated activity in the normal monkey, but phaselocked oscillations appear in the MPTP model of parkinsonism. J Neurophysiol 74:1800-1805.

Olszewski J (1952) The thalamus of the Macaca mulatta. Basel: Karger.

Percheron G, Francois C, Talbi B, Yelnik J, Fenelon G (1996) The primate motor thalamus. Brain Res Brain Res Rev 22:93-181.

Pessiglione M, Guehl D, Agid Y, Hirsch EC, Feger J, Tremblay L (2003) Impairment of context-adapted movement selection in a primate model of presymptomatic Parkinson's disease. Brain 126:1392-1408.

Pessiglione M, Guehl D, Hirsch EC, Feger J, Tremblay L (2004a) Disruption of self-organized actions in monkeys with progressive MPTP-induced parkinsonism. I. Effects of task complexity. Eur J Neurosci 19:426-436.

Pessiglione M, Guehl D, Jan C, Francois C, Hirsch EC, Feger J, Tremblay L (2004b) Disruption of self-organized actions in monkeys with progressive MPTP-induced parkinsonism. II. Effects of reward preference. Eur J Neurosci 19:437-446.

Raz A, Vaadia E, Bergman H (2000) Firing patterns and correlations of spontaneous discharge of pallidal neurons in the normal and the tremulous 1-methyl-4-phenyl-1,2,3,6-tetrahydropyridine vervet model of parkinsonism. J Neurosci 20:8559-8571.

Schneider JS, Kovelowski CJ (1990) Chronic exposure to low doses of MPTP. I. Cognitive deficits in motor asymptomatic monkeys. Brain Res 519:122-128.

Schneider JS, Rothblat DS (1996) Alterations in intralaminar and motor thalamic physiology following nigrostriatal dopamine depletion. Brain Res 742:25-33.

Tremblay L, Filion M, Bedard PJ (1989) Responses of pallidal neurons to striatal stimulation in monkeys with MPTP-induced parkinsonism. Brain Res 498:17-33.

Vitek JL, Ashe J, DeLong MR, Alexander GE (1990) Altered somatosensory response properties of neurons in the "motor" thalamus of MPTP treated parkinsonian monkeys. Soc Neurosci Abstr 16:425.

Vitek JL, Ashe J, DeLong MR, Alexander GE (1994) Physiologic properties and somatotopic organization of the primate motor thalamus. J Neurophysiol 71:1498-1513.

Wichmann T, Bergman H, Starr PA, Subramanian T, Watts RL, DeLong MR (1999) Comparison of MPTP-induced changes in spontaneous neuronal discharge in the internal pallidal segment and in the substantia nigra pars reticulata in primates. Exp Brain Res 125:397-409. 